\title{
Study on Mode of Multi Programming Environment for High Efficiency Machine Simulation System
}

\author{
Yuedong Xiong \\ Department of Precision Manufacture Engineering, Suzhou Institute of Industrial Technology, \\ Suzhou, Jiangsu, China, 215104 \\ xiongyd@siit.edu.cn
}

\begin{abstract}
Keywords: multi programming environment; high efficiency machining; rotational indexing machining; simulation system

Abstract. Through the deep research on the mathematical model of high efficiency machining movement, the method of developing the simulation system of high efficiency machining machine tools in general software environment is discussed. According to the characteristics of high efficiency machining machine tool, the machine simulation system was constructed under multi programming environment through the integration and sharing of computational software, 3D modeling software and platform software function, and the machining simulation was realized. The simulation results not only verify the feasibility of the machining principle, but also prove the stability and effectiveness of the machining simulation system based on the multi programming environment.
\end{abstract}

\section{Introduction}

The high-efficiency is important development direction for NC technology; it is same for technology of manufacturing. Efficient machining by enterprises attaches great importance to, and engineering and technical personnel in the industry devoted a lot of effort in order to improve the efficiency of $\mathrm{CNC}$ machining. Among the many ways to improve the efficiency of CNC machining, there is a method be attention to by researchers that a traditional intermittent machining process becomes a method of continuous process, increase the effective cutting time, shorten the auxiliary processing time; this is the rotational indexing technology. In the process of continuous rotary indexing, processing becomes intermittent movement for continuous processing, to achieve a high-speed continuous cutting. This technology greatly improves the processing efficiency and quality of parts, reducing production costs and improves product competitiveness respond quickly to market. At present rotational indexing processing technology has made great progress, commercialization rotational indexing machine tools has emerged. But the development of the simulation technology of the machine tool is not far better. Researchers in the corresponding field of machining simulation also done some exploration, but the response time is slower on a single software platform developed simulation system, due to the computational complexity; and multi-module simulation system using multiple software development tools, developed a since the programmer did not organically integrate various software, so each module interface has not done seamlessly, resulting simulation system has some shortcomings, the system is not stable enough. So far, there is not a mature system for rotational indexing motion simulation[1,2,3]. To solve this problem, after working on the principle of rotational indexing machining and its simulation conducted in-depth research, this paper attempts to use the same company (for example, PTC Corporation) of the 3D modeling software and computing software to build the machining simulation system, has received very good results.

\section{Rotational indexing machine motion mathematical model}

Rotational indexing machining is the uses of inner-cycloid generate toolpaths to machine the workpiece, so this method is also known as the inner-cycloid processing method[4,5,6,7,8]. As shown in Figure 1 is a inner-cycloid trajectory line occurs, cycloid base circle radius R, a drive circle of radius $\mathrm{r}$; drive circle clockwise from the position I do pure rolling, assuming a disk is fixedly 
connected to a point $\mathrm{P}$ on the rigid body is occurred hypocycloid trajectory, from occurring to the circle center point $\mathrm{P}$ is e, the angle between the cycloidal trajectory start point position and $\mathrm{X}$-axis is $\beta$, occurs do pure drive circle, when rolled position II, the occurrence of self-circle angle is $\theta$, male corner (position I and position II the angle) is a; then the circle occurs during scrolling across the track at point $\mathrm{P}$ can be as follows (1) the expression:

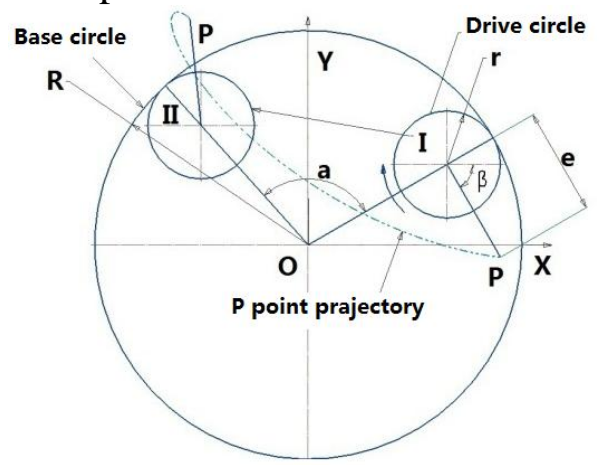

Fig.1 Arbitrary starting position of the cycloid generated within the track

$$
\left\{\begin{array}{l}
x=(R-r) \cos a+e \cos (\theta+\beta) \\
y=(R-r) \sin a+e \sin (\theta+\beta)
\end{array}\right.
$$

Where: $\quad \theta=(1-R / r) a \quad a \geq 0^{\circ}$

As figure 2 shown, if the occurrence of the circle in the horizontal position, $\mathrm{P}$ point on the rigid body in the $\mathrm{X}$-axis, cycloidal trajectory starting angle is zero, when the occurrence of the circle from the position I to scroll to position II, the locus of points P 2 may simplifies to (2) the formula:

$$
\left\{\begin{array}{l}
x=(R-r) \cos a+e \cos \theta \\
y=(R-r) \sin a+e \sin \theta
\end{array}\right.
$$

(1) and (2) is cycloid equation. $a, \theta, R, r$ as a parameter.

Further, if $\mathrm{R}=2 \mathrm{r}$, then the cycloid parametric equation becomes elliptic equation parameters, namely:

$$
\left\{\begin{array}{l}
x=(r+e) \cos a \\
y=(r-e) \sin a
\end{array}\right.
$$

By cycloid parametric equation can be seen: the parameter $\mathrm{R}, \mathrm{r}$ and e will affect the shape of the cycloid change these parameters, cycloid trajectory will change; and the parameter $\beta$ only affects the position of the cycloid occurred.

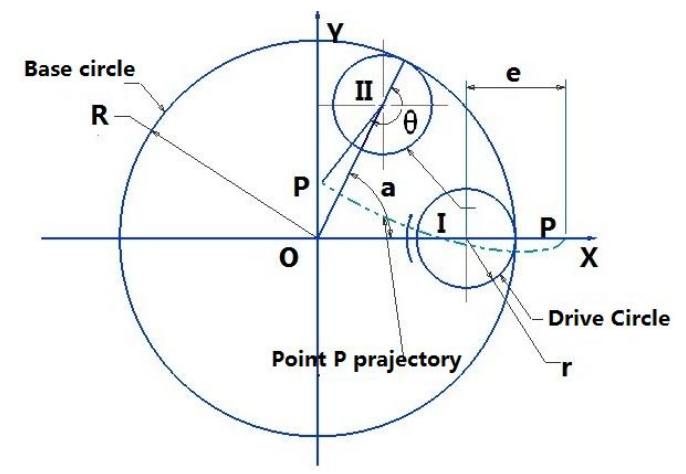

Fig.2 Cycloid generated trajectory of starting Angle 00 


\section{Rotational indexing processing principles}

Shown in Figure 3, we can see that the movement is actually a spin points processed synthetic two movements: one occurs around the base circle round the heart 0 revolution speed of $\mathrm{np}$; the second is the occurrence of a circle around their center of rotation c, the speed of nc. Since the occurrence of the circle along the base circle is made of pure rolling planetary motion, so the occurrence of a fixed point $\mathrm{P}$ on the circle movement indeed, occurred around the same time and the base circle center circle center $\mathrm{C} 0$ planetary motion, its revolution and rotation speed ratio $\mathrm{np}$ : $\mathrm{nc}=\mathrm{r}$ : R. According to the principle of relative motion, without changing the status of the base circle and the relationship between the occurrence of the circular movement, and the occurrence of the base circle center circle center remain fixed point on the circle with respect to the occurrence of the base circle is still cycloidal trajectory $[9,10,11]$.

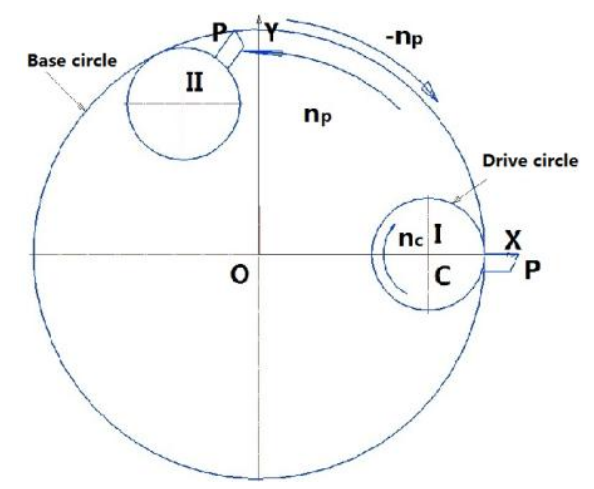

Fig.3 The cycloid machining motion synthesis

Judging from the perspective of spatial kinematic processing spin points, its process is to control the relative position and relative movement of the workpiece and the instantaneous processing of each tool. Ordinary lathe is the workpiece rotation, tool feed; general milling cutter is rotated, the workpiece stationary (or feed). The sub-processes in the application of the principle of spin molding, the tool is rotated, and the work also rotates. By setting the tool and the workpiece at a specific speed than synchronous rotary indexing, so that the tip relative to the surface to be machined out of the hypocycloid trajectory, variable intermittent motion processing for continuous processing campaign to improve processing efficiency, this is the spin points processing Fundamentals.

\section{Build rotational indexing processing simulation system}

Spin sub machining simulation system is to take advantage of multiple generic software modules for the different characteristics of the simulation system, adopt hybrid programming strategy to build $[12,13,14]$. Through the development of generic software modules for each data exchange and seamless connectivity to each software repository organically integrated, quick access to all the advantages of a common software dedicated resources to achieve spin sub-process simulation. The software is mainly responsible for the modeling tool to calculate the trajectory of the workpiece processing systems and operations as well as the logical relationship between them. For process modeling, there is a very good CAD software on the market, such as Pro / E, UG, CATIA, etc.; these applications have powerful modeling capabilities and have a good mechanism for external calls, no longer need to use OpenGL or D3D to carry out a large number of low-level design. For numerical 
problems, but also on the market mature application software, such as MathCAD, Matlab, Mathematica and other numerical software, they have a very powerful computing capability, and there is also a variety of external invocation, these computing tasks can be completed separately. How to choose a suitable mate calculation software and CAD software to build a good finish to become stable, the key sub-process simulation system of rotating seamless process simulation.

By matching and screening CAD software and computing software, eventually found, with the US Company's CAD software, PTC Pro / E and calculation software MathCAD can be done seamlessly. As shown in Figure 4 is a MathCAD dedicated to solving, analysis of industry-standard software and sharing engineering calculations. The software is built hundreds of mathematical functions that can define unlimited personal use of the exclusive function to solve the equation in the form of numbers and symbols, and complex equations. It provides real-time numerical capabilities to quickly build models and interact with other software. More importantly, it can be carried out with the Pro / E seamless connectivity and real-time two-way data transmission, quick calculation results back to Pro / E, to avoid frequent run-time simulation system "card machine" instability phenomenon. Therefore, the spin sub-process simulation system discussed in this article is based on the MathCAD mixed together.

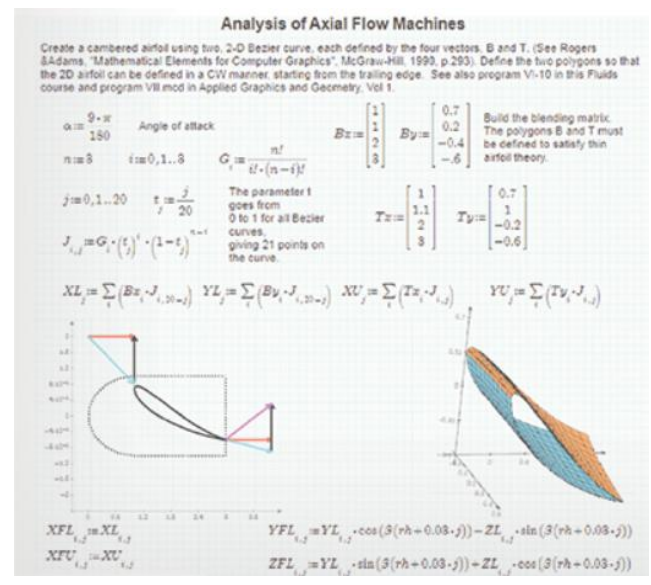

Fig. 4 MathCAD interface

\section{Processing system modeling}

$\mathrm{CNC}$ machine tools, fixtures, tools, and is composed of a rotating workpiece processing sub-system which entities are used Pro / E create; the use of Pro / E and processed in accordance with the above sub-spin motion model, designed as shown as figure 5 concept machine. The machine consists of three translational axes and two rotary axes, namely:

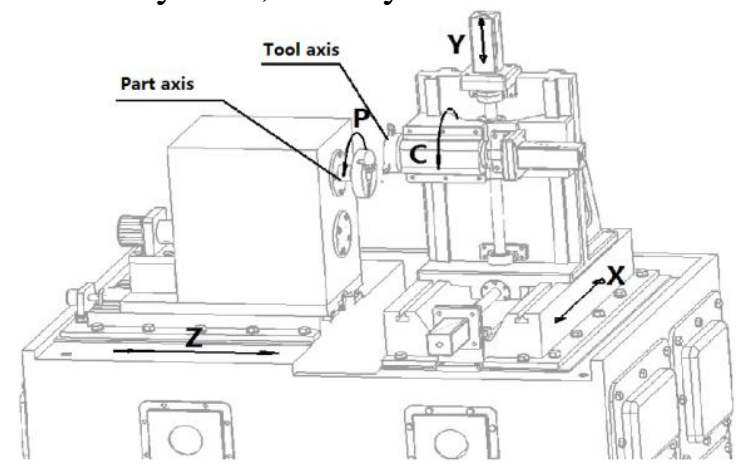

Fig.5 schematic cycloid processing machine concepts 
$\mathrm{X}$-axis perpendicular to the horizontal axis of the tool axis;

Y-axis perpendicular to an axis perpendicular to the axis of the tool;

$\mathrm{Z}$-axis parallel to the horizontal axis of the tool axis;

$\mathrm{P}$ a workpiece axis, perpendicular to the XY plane;

$\mathrm{C}$ a tool axis, perpendicular to the XY plane;

$\mathrm{X}, \mathrm{Y}, \mathrm{Z}$ axes orthogonal to each other, constitute the machine coordinate system, is used to determine the relative position of the tool and the workpiece in the machining process. The tool axis is always parallel to the axis of the workpiece spindle axis, and is perpendicular to the XY plane.

In the rotational indexing machining simulation system is the workpiece (or blank) and the fixture should change with the size of the change process, how to achieve parametric design of the workpiece and fixture, which is built off of the entity model. In the Pro / E environment, use one of the secondary development tool module Toolkit by calling Pro / Toolkit library functions, external programs written using the asynchronous mode (Asynchronous Mode), you can achieve the workpiece and fixture design parameters. The use of the tool is limited, can advance the use of Pro / E software built to a magazine managed by the tool manager shown in Figure 6, to call the library through various tool parameters.

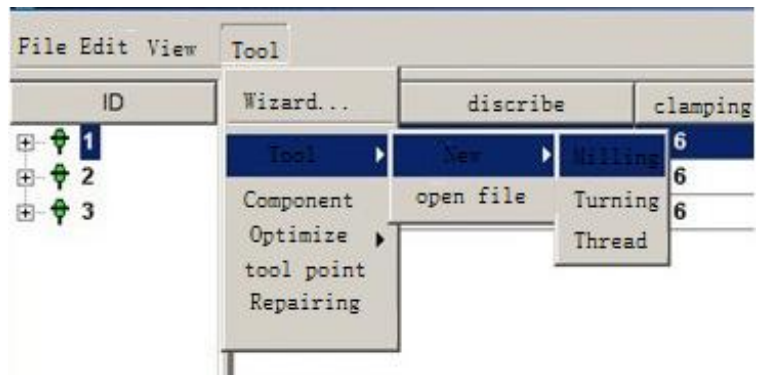

Fig.6 Tool manager interface

\section{Building simulation system}

The simulation system is the use of object-oriented programming language VC ++ and PTC's two software Pro / E and MathCAD established. Model Pro / E construct systems necessary tools, fixtures, tools and the workpiece, etc.; MathCAD determine the various components in the machine in the correct position, speed and tip trajectory parameters and other parameters through $\mathrm{VC}++$ build system control module. On the one hand the system calls the class function and model the dynamic link library Pro / E provided by the control module, the model in real-time editing. On the other hand the use of the master module via the Pro / E Toolkit development tools MathCAD class function calls is calculated in real time and the results are returned to the calling module, enabling simulation of spin-minute process.

\section{Machining simulation}

Shown in Figure 7, the next user graphical user interface spin points in machining simulation system, by way of human-computer interaction, and easily call the model library spin sub-machine tools and related equipment in the computer technology, machine polygonal body and sharp teeth gear. Such as polygon turning or rotating polygonal body on the body, can make (1), (2) where the parameter $\mathrm{R}=\mathrm{kr}$. 


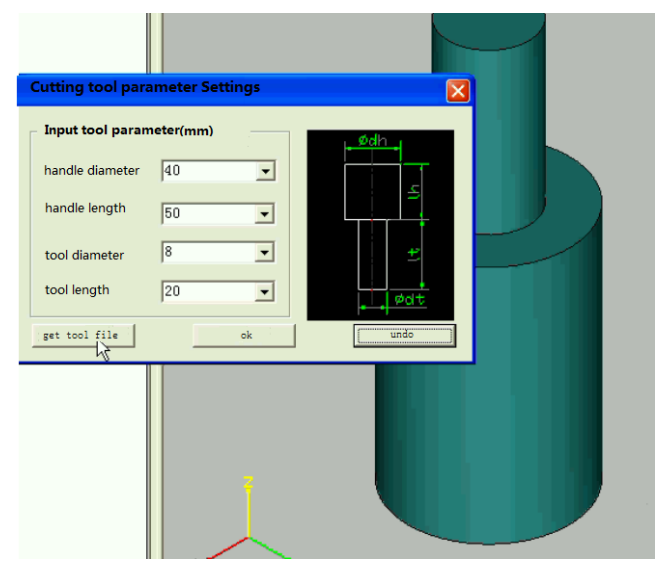

Fig.7 The graphical interface machining simulation system

When $\mathrm{k}=2$ 时, $\mathrm{R}=2 \mathrm{r}$, cycloid equation becomes elliptic equations, represented by (3). Rotation of the tool in the rotating workpiece across the track shown in Figure 8, the processing methods used to process the end face straight slot or cross slot.

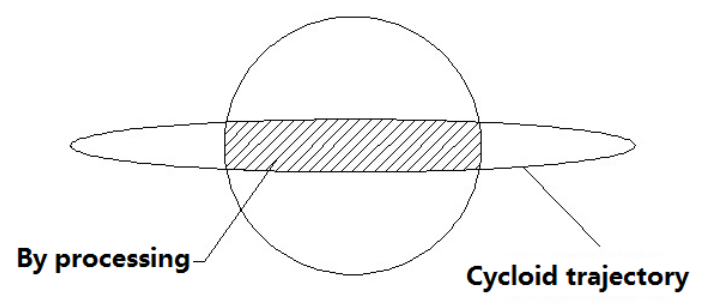

Fig.8 Point across elliptical locus

When $\mathrm{k}=3,4,5,6$, the can be processed separately on a rotator regular triangle, a regular tetragon, pentagon, hexagon, etc., processing is hexagonal obtained for computer simulation shown in Figure 9 shape results.

When processing, polygon processing methods can be carried out. In order to improve production efficiency, can be processed in multiple carbide blade cutter body tops, the number of teeth on the cutter body is most desirable processing half the number of teeth.

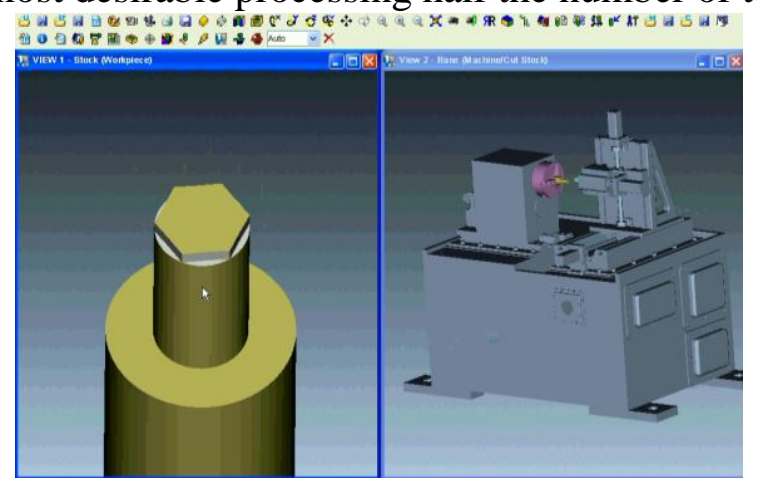

Fig.9 Hexagon processing simulation

During the study, by Pro / E, respectively, MathCAD, Matlab, Mathematica and other numerical software paired constitute three systems, and process simulation comparison experiments. Using $\varphi 25$ $\times 50 \mathrm{~mm}$ round rod in the experiment, it is required to be processed into a side length of a straight line is less than or equal to $0.03 \mathrm{~mm}$ regular hexagon. The results showed that: the simulation system consists of MathCAD and Pro / E component, there is no "card machine" phenomenon during the simulation, process simulation takes the shortest, only nine seconds. Compare machining simulation results shown in Table 1. 
Table1: Machining simulation experimental comparison

\begin{tabular}{|c|c|c|}
\hline $\begin{array}{l}\text { Numerical calculation softwar } \\
\text { e to pair with Pro/E }\end{array}$ & $\begin{array}{l}\text { Machining process simulation t } \\
\text { ime consuming }\end{array}$ & Be stuck \\
\hline MathCAD & 9 & $\mathrm{~N}$ \\
\hline Matlab & 18 & $\mathrm{Y}$ \\
\hline Mathematica & 16 & $\mathrm{Y}$ \\
\hline
\end{tabular}

\section{Conclusions}

By rotating sub-process mathematical modeling process, analyzes the basic principle of this processing technology; accordingly proposed mixed development model based on MathCAD, spin sub-process simulation system was constructed on a multi-platform software development. This method makes full use of PTC's two software (PRO / E and MathCAD) exclusive resources and good compatibility and seamless connectivity features between them, they are organically integrated into a simulation of the whole, so called the two software simulation systems perform their duties effectively play its due role for the simulation system to provide rapid response in human-computer interaction behavior. In this paper, a simulation example demonstrates the stability, effectiveness and practicality of the system. Apply this simulation system for verification before the actual CNC machining tool path and processing procedures and shorten the manufacturing cycle, reducing manufacturing costs and improve production efficiency. No doubt, a mixed development MathCAD based approach provides a way to quickly and effectively develop simulation system for such programmers.

\section{References}

[1] Peng Gao: in Tianjin university, 2006

[2] Jianghua He: in Tianjin university, 2007

[3] Yuedong Xiong, Hong Gao, Zuquan Zhang: in Coal Mine Machinery, 2012(6)

[4] Xiliang Liu, Xianghong Liu: in Journal of Changsha Railway Institute, 1998 .16(1)

[5] Jibo Liu: in Mechanical Transmission, 1992.19 (3)

[6] Baomin Wang: in Mechanical Science and Technology, 2002.21 (1)

[7] Xiaoyan Xia,: in Automobile Gear Technology, 2002 (1)

[8] Jia Li, Jianghua He: in Journal of Mechanical Engineering, 2008 (5);

[9] Yuedong Xiong, Jiandong Cao, Xi Chen: in Modular Machine Tool \& Automatic Manufacturing Technique, 2011(11)

[10]Limin Dong, Zhicang Zhang, Yuedong Xiong: in Modular Machine Tool \& Automatic Manufacturing Technique, 2007(12)

[11] Yanbo Zhang: in Combination Machine Tools and Automatic Processing Technology, 2003 (4)

[12] Jia Li, Peng Gao, Jianghua He: in China Mechanical Engineering, 2007(19)

[13] Yongxin Liu: in Wood Processing Machinery. 1999(1)

[14] Wei Zhang, Taiyong Wang, Yuedong Xiong: in Manufacturing Automation. 2006(6) 